\title{
Clinical Feasibility Assessment of T3 Sub-Stage in Rectal Cancer Using
}

\section{MRI}

\author{
Tripathi Pratik, ${ }^{1,2}$ Weifeng Guo, ${ }^{1,2}$ Chun Yang, ${ }^{1,2}$ Rai Bimal, ${ }^{3}$ and Mengsu Zeng, ${ }^{1,2}$, \\ ${ }^{1}$ Department of Radiology, Zhongshan Hospital, Fudan University, Shanghai, China \\ ${ }^{2}$ Shanghai Institute of Medical Imaging, Department of Medical Imaging, Shanghai Medical School, Fudan University, Shanghai, China \\ ${ }^{3}$ Department of Radiology, Tongji Hospital, Tongji Medical College, Huazhong University of Science and Technology, Wuhan, China \\ "Corresponding author: Mengsu Zeng, Department of Radiology, Zhongshan Hospital, Fudan University, Shanghai Institute of Medical Imaging, Department of Medical \\ Imaging, Shanghai Medical School, Fudan University, Shanghai, China, E-mail: zengmengsu@outlook.com
}

Received 2017 March 16; Revised 2018 January 21; Accepted 2018 January 29.

\begin{abstract}
Background: MRI predicted distance of mesorectal extension (mrDME) in rectal cancer is one of the independent risk factors for recurrence and poor overall survival. In T3 rectal cancer, if no lymph node or distant metastasis is seen, the selection of optimum treatment is based on the distance of mesorectal extension. Therefore, it is very crucial to investigate the reproducibility of DME in T3 rectal cancer.

Objectives: To investigate the reproducibility of the distance of mesorectal extension by tumor invasion in T3 stage rectal cancer by evaluating sub-stages T3a, T3b T3c and T3d individually versus T3a, T3b (T3ab) and T3c, T3d (T3cd) combined together using MRI. Patients and Methods: From July 2014 to December 2015, 188 patients with surgically and histologically confirmed T3 rectal cancer who underwent preoperative MRI were enrolled into this study. Two blinded radiologists evaluated the maximum distance of mesorectal extension (mrDME) in T2 weighted image in MRI. The study population was sub classified into T3a $(<1 \mathrm{~mm})$, T3b (1 - 5 $\mathrm{mm})$, T3c (5-15 mm) and T3d ( $>15 \mathrm{~mm}$ ) according to the distance of mesorectal extension by tumor invasion. The inter-observer and intra-observer agreements were then assessed using kappa $(\mathrm{k})$ coefficient of agreement and intraclass correlation coefficient (ICC).

Results: Difference in the value of inter-/intra-observer kappa, and inter-/intra-observer ICC between the two groups was very distinct. In the individual group (T3a, T3b, T3c and T3d), the inter-observer and intra-observer ( $\mathrm{k}$ ) for the mrDME was 0.700 and 0.718 respectively; the inter-observer and intra-observer ICC was 0.772 and 0.786 respectively. In the combined group (T3ab and T3cd), the inter-observer and intra-observer kappa $(\mathrm{k})$ for the mrDME was 0.819 and 0.883 respectively; the inter-observer and intra-observer ICC was 0.829 and 0.796 respectively.

Conclusion: There was a distinct increase in the kappa and ICC value in the combined group compared with the individual group. This high reproducibility result suggested that it is more reliable to measure T3ab and T3cd combined together than individually. This finding can play a crucial role in the management of rectal cancer and clinical decision making for non-expert radiologists in non-academic setting.
\end{abstract}

Keywords: Rectal Cancer, T3 Sub-Staging, Reproducibility Test, MRI, Distance of Mesorectal Extension, mrDME

\section{Background}

Rectal cancer is one of the major causes of cancer mortality in the world (1). MRI is considered as the choice of modality for rectal cancer staging as it provides classification of the mesorectal fascia with high accuracy (2-5). MRI is capable of diagnosing rectal cancer very accurately. $\mathrm{T}$ stage (tumor stage) defines the primary tumor site including the depth of tumor invasion. In preoperative T staging of rectal cancer, the reported overall accuracy is $71 \%$ - 91\% (2). Many studies have shown extramural depth of tumor invasion also known as distance of mesorectal extension
(DME) as an independent risk factor for tumor recurrence and overall survival rate $(6,7)$. The depth of tumor invasion is proportional to the survival, most importantly for T3 rectal cancers (7).

T3 tumors penetrate the muscular layer and invade into the subserosa. T3 rectal cancer is sub-classified as T3a, T3b, T3c and T3d if DME is $<1 \mathrm{~mm}, 1-5 \mathrm{~mm}, 5-15 \mathrm{~mm}$ and $>15 \mathrm{~mm}$ respectively. The extramural depth of tumor invasion for T3a $(<1 \mathrm{~mm})$ on MRI is too small for measurement and can vary from person to person. Because the distance is very narrow, therefore, the diagnosis might frequently differ from person to person that finally results in the differ- 
ent treatment method. One of the studies suggested that with respect to a 5-year disease free survival rate, there was no significant difference between T3a and T3b or T3c and T3d, but was statistically significant when re-categorized as T3ab and T3cd using $5 \mathrm{~mm}$ as cutoff point (8). Many previous studies investigated the prognosis of T3 subdivision using various cutoff points ( $4 \mathrm{~mm}, 5 \mathrm{~mm}$, and $6 \mathrm{~mm}$ ) and came to conclusion that the deeper the tumor invasion, the worst the prognosis $(9,10)$. Recently, using $5 \mathrm{~mm}$ as a cutoff point to differentiate early and advanced $\mathrm{T} 3$ rectal cancer is highly acceptable. According to recent studies, patients with a DME more than $5 \mathrm{~mm}$ should be identified properly because they have a worse prognosis compared to patients with DME less than $5 \mathrm{~mm}$ (11).

In a non-academic setting, non-expert readers evaluate many rectal cancer patients; therefore, in order to avoid over- and under-treatment, a high reproducibility is very crucial. The aim of this study was to categorize the four sub-stages (T3a, T3b, T3c and T3d) into two sub-stages (T3ab, and T3cd) in T3 staged tumor i.e. early-staged T3 tumor ( $\mathrm{mrDME}<5 \mathrm{~mm})$ and advance-staged T3 tumor (mrDME $>5 \mathrm{~mm}$ ) to check the reproducibility between the two groups. Because of the narrow measurement limitation of T3a tumor, it proves to be very challenging for non-expert radiologists and may result in varying $\mathrm{T} 3$ stage even by expert radiologists.

\section{Objectives}

To investigate the reproducibility of the distance of mesorectal extension by tumor invasion in T3 stage rectal cancer by evaluating sub-stages T3a, T3b, T3c, and T3d individually versus T3a, T3b (T3ab), T3c, and T3d (T3cd) combined together using MRI.

\section{Patients and Methods}

Between July 2014 and December 2015, 314 consecutive patients who were diagnosed with rectal cancer using MRI and confirmed by post-surgical pathological test were included in this study. The patients underwent curative surgery and post-surgical pathological test for rectal cancer at Zhongshan hospital affiliated to the Fudan University. The post-surgical pathological test confirmed the tumors as T3 tumors but sub-staging of T3 was not done. Data such as extramural venous invasion (EMVI), nodal status, and location of tumor were also collected but was not used for this specific study. Patients $(n=126)$ with the following characteristics were excluded from this study: pathological T1/T2/T4 $(n=101)$, incomplete MRI data $(n=11)$, preoperative chemo-radiotherapy ( $\mathrm{n}=9)$, and history of other cancer diagnoses $(n=5)$. One hundred eighty-eight patients who were confirmed as $\mathrm{T} 3$ stage rectal cancer by the pathological diagnosis were only included in this research. High, mid, and low rectal cancer was categorized according to the location of the tumor $(0-6 \mathrm{~cm}$, lower rectum; 7-11 cm, middle rectum; and $12-15 \mathrm{~cm}$, upper rectum) (12). Invasion of the surrounding soft tissue through the external sphincter, but with no other organ invasion were also included in this study and considered as lower T3 rectal tumor (13). After exclusion, we had complete data available for analysis of 188 patients with clinical T3 rectal cancer. The entire process is as shown in the flowchart (Figure 1) and patient demographics characteristics are shown in Table 1.

\begin{tabular}{|c|c|c|}
\hline Characteristics & Frequency, No. & $\%$ \\
\hline \multicolumn{3}{|l|}{ Sex } \\
\hline Female & 59 & 31.4 \\
\hline Male & 129 & 68.6 \\
\hline \multicolumn{3}{|l|}{ Tumor location } \\
\hline High & 18 & 9.6 \\
\hline Low & 84 & 44.7 \\
\hline Middle & 86 & 45.7 \\
\hline \multicolumn{3}{|c|}{ Pathological vascular invasion } \\
\hline Negative & 153 & 81.4 \\
\hline Positive & 35 & 18.6 \\
\hline \multicolumn{3}{|c|}{ Pathological lymphatic invasion } \\
\hline Negative & 103 & 54.8 \\
\hline Positive & 85 & 45.2 \\
\hline \multicolumn{3}{|c|}{ Pathological perineural invasion } \\
\hline Negative & 129 & 68.6 \\
\hline Positive & 59 & 31.4 \\
\hline \multicolumn{3}{|c|}{ Reader1 T3 sub-staging } \\
\hline Тза & 10 & 5.3 \\
\hline T3b & 81 & 43.1 \\
\hline T3c & 92 & 48.9 \\
\hline T3d & 5 & 2.7 \\
\hline \multicolumn{3}{|c|}{ Reader2 T3 sub-staging } \\
\hline Т3а & 2 & 1.1 \\
\hline T3b & 89 & 47.3 \\
\hline $\mathrm{T} 3 \mathrm{C}$ & 94 & 50.0 \\
\hline T3d & 3 & 1.6 \\
\hline
\end{tabular}

${ }^{\mathrm{a}}$ The distribution of general population and the readings carried out by the two readers are shown in the table above. The obvious difference in T3a can be seen between the two readers. 


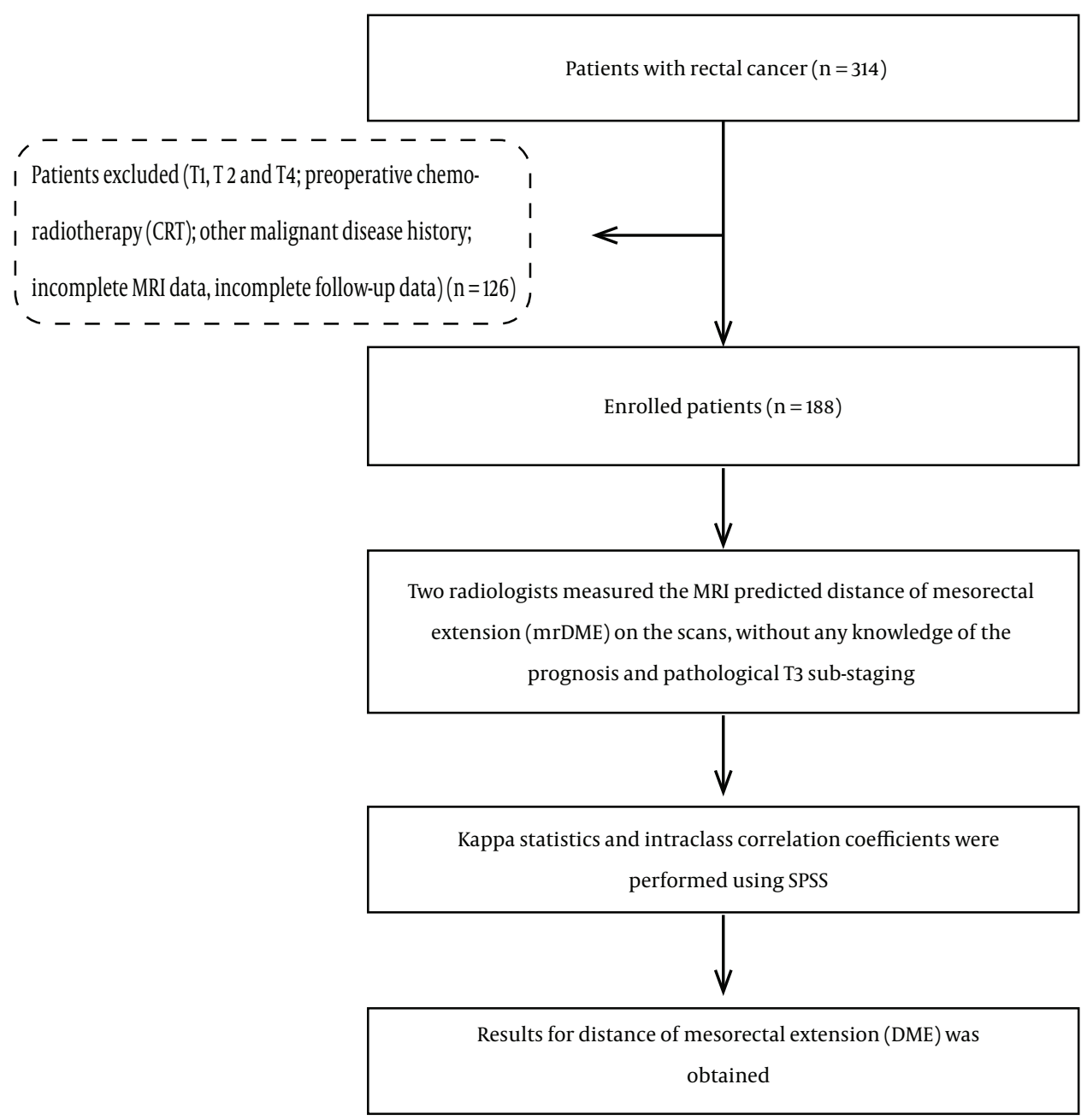

Figure 1. Flowchart of patints' enrollment, data collection and analysis.

\subsection{MRI Method}

One hundred and eighty-eight patients with primary T3 rectal cancer were assessed by routine coronal, axial, and sagittal MRI at 1.5 or $3.0 \mathrm{~T}$, including T1 weighted image (T1WI), T2 weighted image (T2WI), apparent diffusion coefficient (ADC), diffusion weighted image (DWI), and contrast image. Two radiologists with 12 and 10 years of abdominopelvic MRI reporting experience measured the MRI-predicted DME (mrDME) on the scans, without any knowledge of the prognosis and pathological T staging. All images were evaluated using PACS workstation. Presence or absence of any distant metastasis at imaging and surgery was recorded.

The rectal MRI examinations were done using three different MRI machines (1.5 T Siemens Avanto, 1.5 T Siemens
Aera, and 3.0 T Seimens Verio, Siemens Medical Systems, Erlangen, Germany) in our institution. Axial T2 weighted image without fat saturation was used for extramural vascular examination. The MRI protocol in our institution consisted of the following sequences in Table 2. Sagittal T2 weighted image without fat saturation also used the same imaging protocol with the exception that the plane alignment through which images were taken were changed manually according to the location of the tumor.

\subsection{Magnetic Resonance Distance Predicted Mesorectal Exten- sion Criteria (mrDME)}

The radiologists used $\mathrm{T} 2$ weighted image for the measurement of mrDME and it was performed using the method Miyoshi et al. used. Each reader measured the dis- 


\begin{tabular}{|c|c|c|c|}
\hline \multirow[t]{2}{*}{ Sequence Key Features } & \multicolumn{3}{|c|}{ Sagittal T2 TSE } \\
\hline & VERIO 3.0 T & AVANTO 1.5 T & AERA 1.5 T \\
\hline Repetition time, ms & 3840 & 3000 & 5740 \\
\hline Echo time, ms & 89.0 & 97 & 82.0 \\
\hline Number of slices & 24 & 20 & 25 \\
\hline FOV, mm & 280 & 200 & 280 \\
\hline Base resolution & 320 & 320 & 320 \\
\hline Slice thickness, mm & 5 & 4 & 4 \\
\hline Phase FOV, \% & 73.8 & 100 & 100 \\
\hline Distance factor, \% & 30 & 0 & 30 \\
\hline Phase encode direction & $A>>P$ & $A>>P$ & $\mathrm{H}>>\mathrm{F}$ \\
\hline Phase resolution, \% & 70 & 85 & 70 \\
\hline
\end{tabular}

Abbreviations: FOV, field of view; T, Tesla; T2 TSE, T2-Weighted turbo spin-echo (TSE).

${ }^{\mathrm{a}}$ Three different MRI machines were used for the pelvic imaging. No different machines were used in the same patients for both readers.

tance from the outer extreme point of the muscular layer to the most protruded part in the mesorectal layer. The deepest tumor invasion point in serosa was identified. An imaginary line was drawn between two edges of the muscular layer to estimate the outer border if the outer border of the muscular layer was not clearly identified. A dotted line was drawn in case of difficulty in identifying the exact outer border $(10,14)$ (Figures 2 and 3 ). Reader 1 repeated the same process thrice and the mean value was obtained. The time interval for repetition of the process by reader 1 was set as two weeks. Reader 2 performed it only once.

\subsection{Statistical Analysis}

SPSS (version 21.0, Inc., Chicago, IL) was used to perform statistical analysis. Cohen's kappa and the intraclass correlation coefficients (ICC) two-way absolute agreement method were computed to compare the readings. The first reading by reader 1 was used to evaluate inter-observer analysis. The mean value of 2nd and 3rd observations was obtained. We then categorized the mean value thus obtained into stages T3a, T3b, T3c and T3d, according to the depth of mesorectal extension (T3a $=0-1 \mathrm{~mm}, \mathrm{~T} 3 \mathrm{~b}=1-5$ $\mathrm{mm}, \mathrm{T} 3 \mathrm{c}=5-15 \mathrm{~mm}, \mathrm{~T} 3 \mathrm{~d}=>15 \mathrm{~mm}$ ), which was used for intra-observer analysis. The criteria used for inter-/intraobserver agreements of mrDME measurements using Cohen's kappa and ICC were set as follows: (Kappa statistics $(\kappa)$ ranging from -1 to 1 , with 1 indicating perfect agreement, 0 indicating completely random agreement, and -1 indicating "perfect" disagreement. Values from 0.0 to 0.2 indicate slight agreement, 0.21 to 0.40 indicate fair agreement, 0.41 to 0.60 indicate moderate agreement, 0.61 to
0.80 indicate substantial agreement, and 0.81 to 1.0 indicate almost perfect or perfect agreement. Similarly, ICCs values less than 0 indicate no agreement, 0 - 0.20 as poor correlation, $0.21-0.40$ as fair, 0.41 - 0.60 as moderate, 0.61 - 0.80 as substantial, and $0.81-1$ as excellent) $(15,16)$. We also performed Bland-Altman analysis between two readers (Figure 4). The mean and difference between two readers were obtained. We performed one sample T test. The $P$ value for one sample $T$ test was obtained which was not statistically significant. So we further calculated upper and lower 95\% confidence intervals. Bland-Altman plot was obtained. Finally, we ran a linear regression test to check whether the data had any potential bias. A statistically nonsignificant $\mathrm{P}$ value $(\mathrm{P}>0.05)$ meant there was no proportional bias.

\section{Results}

\subsection{Demographics}

Totally, 188 patients were enrolled in this study. The mean age of the patients was $60.99 \pm 11.88$ years, $68.6 \%$ were male and $31.4 \%$ were female. $9.6 \%, 45.7 \%$ and $44.7 \%$ of the tumors were found in the high, middle, and low rectal, respectively. The positive pathological vascular invasion, lymphatic invasion and perineural invasion were $81.4 \%, 54.8 \%$, and $68.6 \%$, respectively. The result for $\mathrm{mrDME}$ from reader 1 showed 5.3\%, 39.9\%, 52.1\%, and $2.7 \%$ for T3a, T3b, T3c, and T3d, respectively; reader 2 showed $1.6 \%, 44.7 \%$, $52,1 \%$, and $1.6 \%$ for T3a, T3b, T3c, and T3d, respectively.

\subsection{MRI Distance of Mesorectal Extension ( $m r D M E)$}

Mean mrDME for readers 1 was $5.813 \pm 3.13 \mathrm{~mm}$ and for reader 2 was $5.930 \mathrm{~mm} \pm 2.97 \mathrm{~mm}(\mathrm{P}<0.05)$. The interobserver result after analyzing T3a, T3b, T3c and T3d individually, value for $\mathrm{k}$ was 0.700 and for the intra-observer, the value of $\mathrm{k}$ was 0.718 (both substantial agreement); the inter-observer intraclass correlation coefficient ICC was 0.772 (95\% Cl 0.707 - 0.824) and intra-observer intraclass correlation coefficient ICC was 0.786 (95\% Cl 0.725 - 0.835). For T3ab and T3cd after combined together the inter-observer value for $\mathrm{k}$ was 0.829 (almost perfect agreement) and for intra-observer study the value of k was 0.796 . The inter-observer intraclass correlation coefficient ICC was 0.829 (95\% Cl 0.778 - 0.869) and intra-observer intraclass correlation coefficient ICC was 0.796 (95\% Cl 0.737 0.843).

\subsection{ICC Between Two Groups in Two Readers}

The overall agreement between two readers increased from 0.772 to 0.829 (Table 3). The inter-observer kappa (k) increased from 0.700 to 0.819 (Table 4 ). The value above 0.8 is considered as almost perfect and can be used clinically. 

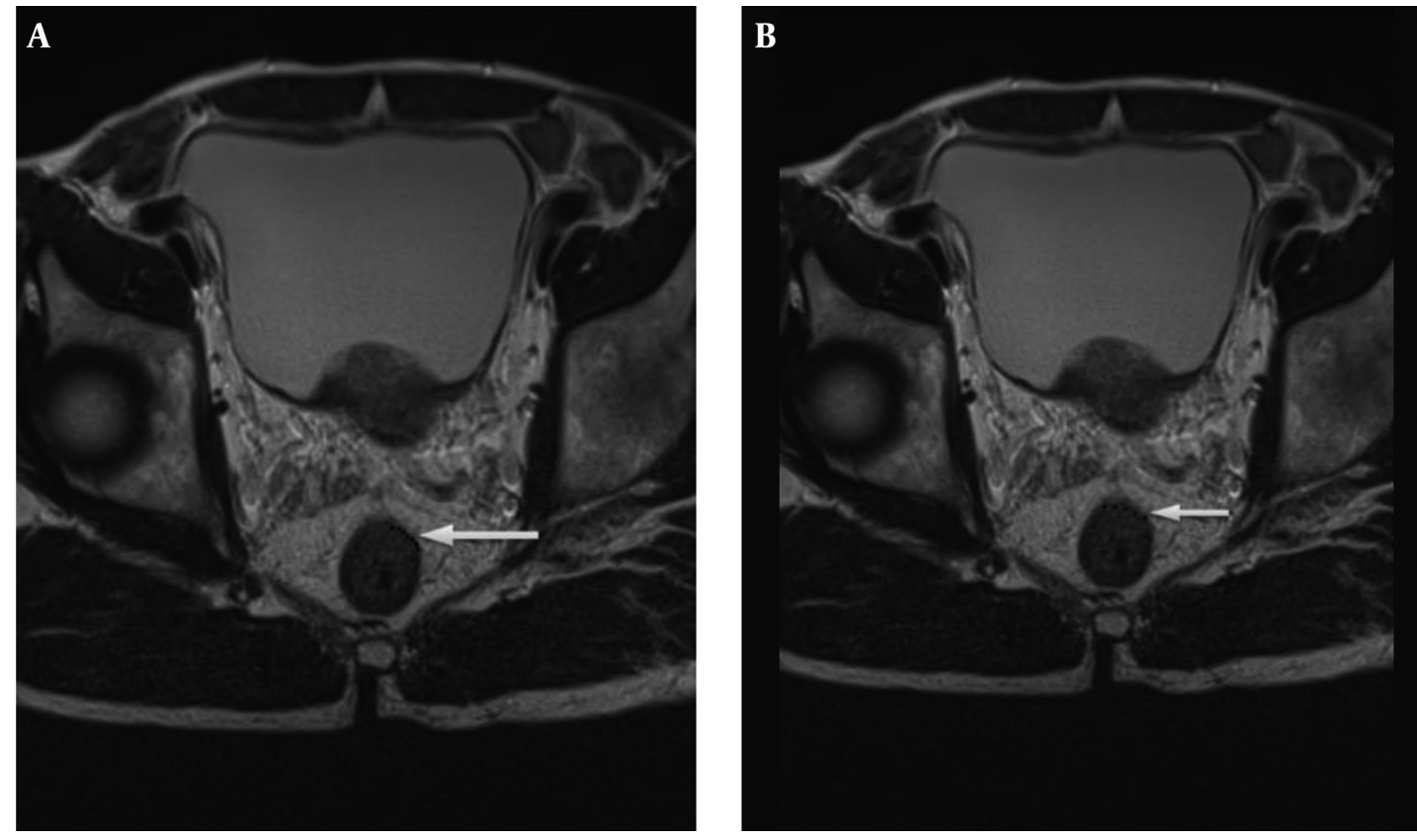

Figure 2. Tumor extension is seen beyond muscularis propria (hypointense signal in T2WI). A, The MRI-predicted distance of mesorectal extension (mrDME) measurement of the T3 rectal cancer by reader 1 was $0.81 \mathrm{~mm}$ (T3a), the area is shown by the white arrow. B, The mrDME measurement of the T3 rectal cancer by reader 2 was $1.6 \mathrm{~mm}$ (T3b); the white arrow shows the area. The black dots are the boundary from which the readers started their measurement.
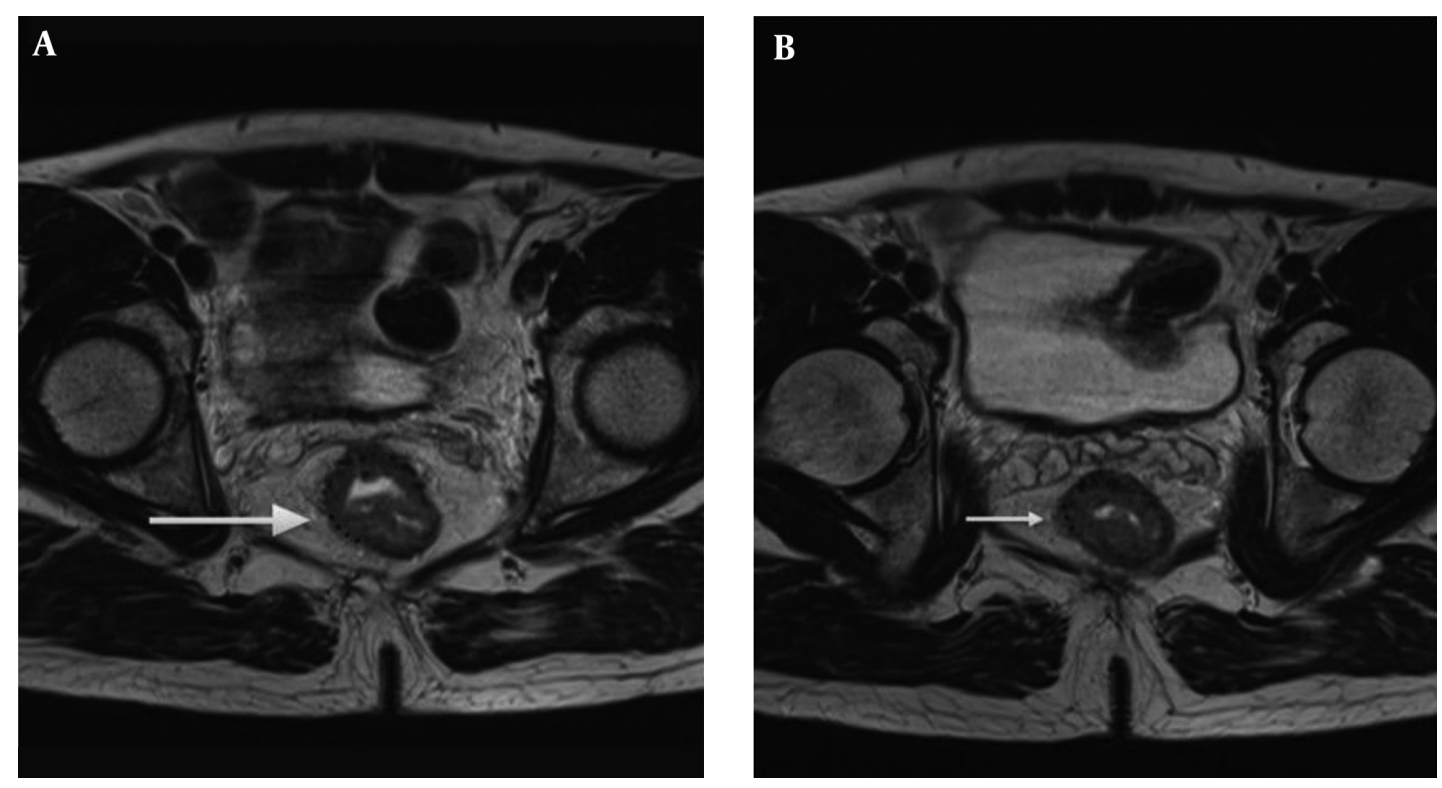

Figure 3. Tumor extension is seen beyond muscularis propria (hypointense signal in T2WI). A, The MRI-predicted distance of mesorectal extension (mrDME) measurement of the T3 rectal cancer by reader 1 was $3.8 \mathrm{~mm}$ (T3b), the area is shown by the white arrow. B, The mrDME measurement of the T3 rectal cancer by reader 2 was $5.1 \mathrm{~mm}$ (T3c); the white arrow shows the area. The black dots are the boundary from which the readers started their measurement.

\section{Discussion}

This study emphasizes the importance of staging T3 rectal tumors into early-staged (T3ab) and advance-staged
(T3cd) rather than T3a, T3b, T3c, and T3d individually. Our study supports the concept of dividing $\mathrm{T} 3$ rectal cancer into 


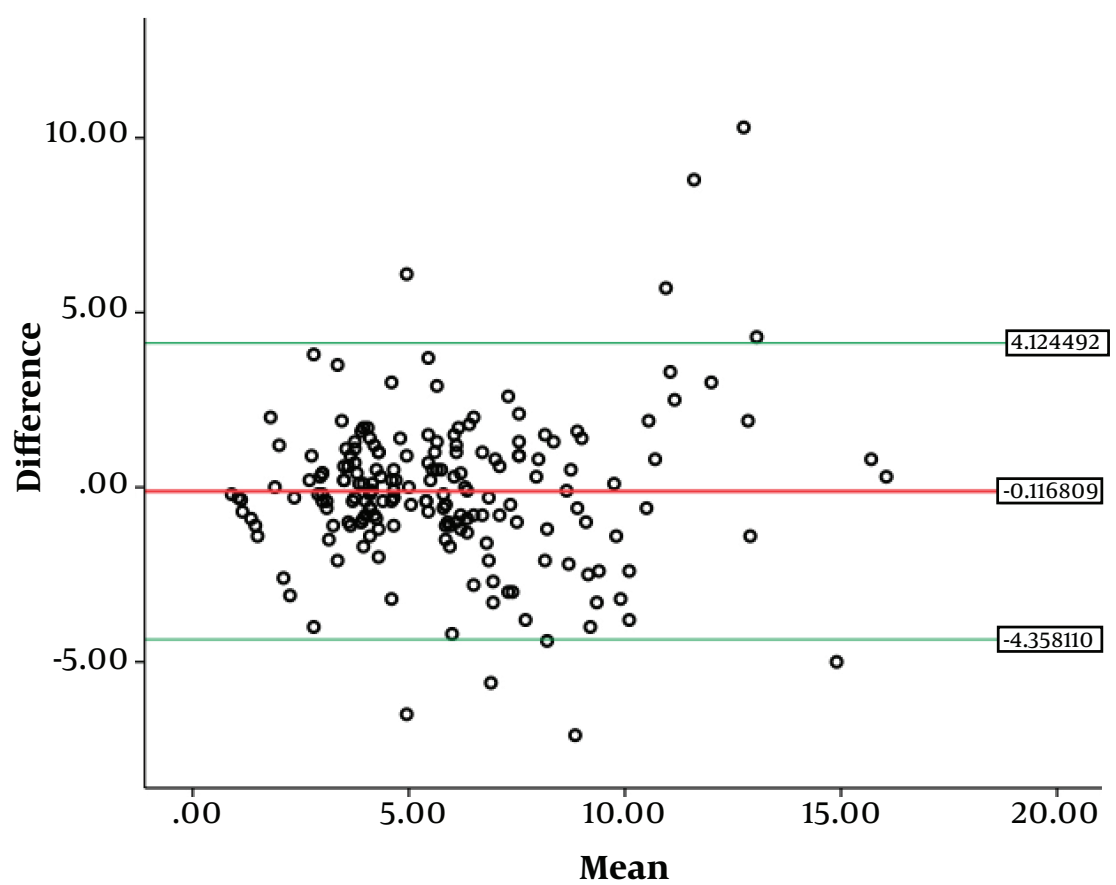

Figure 4. Bland-Altman plot between two readers to show reproducibility in T3 rectal cancer; (note: the red line denotes the mean value, green lines are upper and lowe bounds of $95 \%$ confidence interval)

Table 3. Intra- and Inter-observer Intraclass Correlation Coefficient (ICC) for T3 Rectal Cancer (T3a vs. T3b vs. T3c vs. T3d and T3ab vs. T3cd)

\begin{tabular}{|cccc}
\hline Intraclass correlation coefficient (ICC) & & 95\% Confidence interval & P value \\
\cline { 3 - 4 } & & Lower Bound Bound & 0.823824 \\
\hline T3a vs. T3b vs. T3c vs. T3d & & & 0.835336 \\
Inter-observer & 0.772 & 0.706958 & 0.001 \\
\hline Intra-observer & 0.786 & 0.724954 & 0.001 \\
\hline T3ab vs. T3cd & & & 0.868737 \\
\hline Inter-observer & 0.829 & 0.778038 & 0.001 \\
\hline Intra-observer & 0.796 & 0.736777 & $<0.001$ \\
\hline
\end{tabular}

Table 4. Intra- and inter-observer Kappa (K) for T3 Rectal Cancer (T3a vs. T3b vs. T3c vs. T3d and T3ab vs. T3cd) ${ }^{\mathrm{a}}$

\begin{tabular}{lcc}
\hline Parameter & Inter-Observer Kappa $(\mathbf{k})$ & Intra-Observer Kappa $(\mathbf{k})$ \\
\hline T3a vs. T3b vs. T3c vs. T3d & 0.700 & 0.718 \\
T3ab vs. T3cd & 0.819 & 0.883
\end{tabular}

${ }^{a}$ Obvious increase in the agreement between the two readers after combining two sub-stages can be seen. ICC and kappa ( $\mathrm{k}$ ) both increased after re-classifying four sub-stages into two sub-stages.

early (DME $<5 \mathrm{~mm}$ ) and advanced stage (DME $>5 \mathrm{~mm}$ ) $(7,17,18)$. The inter-observer agreement in our study was almost perfect; some studies have also reported the agreement to be good to very good in the sub-classification of T3 tumors with DME cutoff of $5 \mathrm{~mm}$ on MRimages $(19,20)$. The reproducibility in inter-observer ICC also increased when sub-stages were combined together. The moderate agreement when sub-stages were measured individually can be doubtful in clinical decision-making. The almost perfect agreement result after two sub-stages combined together could be reliable for clinical decision-making. Statistically, the Bland-Altman analysis also supports our hypothesis 
with no proportional bias. By looking at these results, we can say that MRI can be used as a reproducible imaging modality for measurement of DME.

\subsection{Management for T3 Rectal Cancer}

As previous studies have already mentioned, the management and treatment method within T3 tumor differs. Therefore, accurate staging is very essential. The pathological presentation is depicted in Figure 5. According to ESMO clinical practice guidelines for rectal cancer (21), generally the treatment method for T3a and T3b or good group (DME $<5 \mathrm{~mm}$ ) is the same (surgery alone, and radical dissection using TME method), and for T3c and T3d or intermediate/bad group (DME $>5 \mathrm{~mm}$ ), the treatment method is similar (preoperative chemoradiotherapy followed by TME). Management consideration (adjuvant chemotherapy after neoadjuvant chemoradiotherapy/surgery regardless of the surgical pathology results) for T3 tumors as a whole without nodal involvement and even $\mathrm{T} 2$ rectal cancers are found to be the same (22). However, the clinician makes the final decision for the treatment considering the degree of tumor extension and metastasis. The preoperative staging of rectal cancer tumor has been correlated with the patient's disease-free survival and prognosis $(17,22)$. The advantage of pre-operative MRI is that the patients with potentially involved margins can receive neoadjuvant therapy that results in tumor down- staging so that tumor regression can help the follow-up treatment. There is also debate about the treatment method for T3 rectal cancer patients, whether all patients with T3 should undergo pre-operative chemo-radiotherapy (CRT) or surgery alone $(23,24)$. However, this way some patients who might not need the preoperative neoadjuvant therapy undergo unnecessary chemo-radiation. Although this might lessen the probability of tumor regeneration, the health situation beside tumor is severely affected by radiation. Our sub-staging model might be useful in considering management criteria.

\subsection{Re-Categorization of T3 Sub-Staging}

MRI not only helps in the pre-treatment examination and accurate diagnosis but also in the post-treatment analysis. DME with less than $5 \mathrm{~mm}$ has proved to show a significant higher survival rate, the early T3 tumors can be managed with surgery alone, and the prognosis is comparable to T1/T2 tumors (23). A study conducted by Merket et al. in 2001 showed a 5-year survival rate for the early-staged tumor patients DME < $5 \mathrm{~mm}$ (T3ab) was $85 \%$ - 90\% and for advanced tumor DME $>5 \mathrm{~mm}$ (T3cd) was only $54 \%$. Many other studies analyzed the impact of extramural spread (< $5 \mathrm{~mm}$ and $>5 \mathrm{~mm}$ ) with respect to the survival rate in patients with no neoadjuvant chemo therapy or preoperative

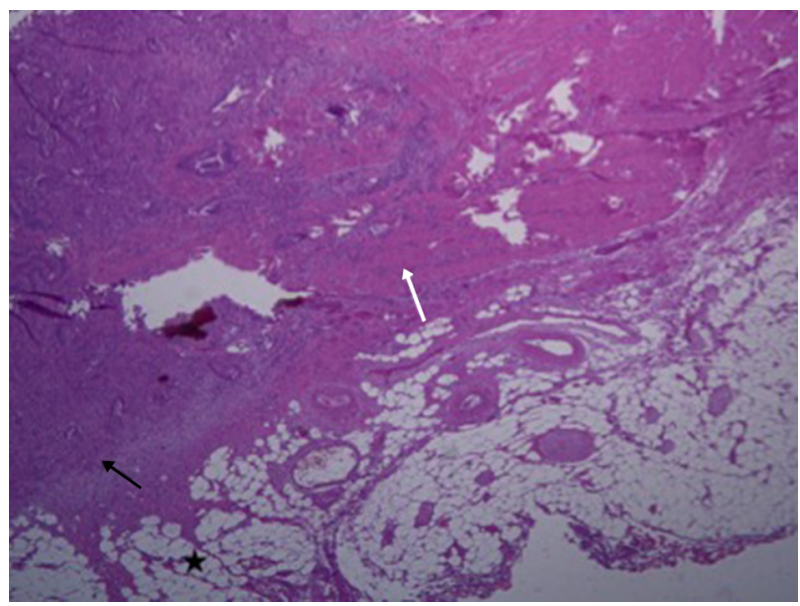

Figure 5. Tumor extension (histopathology). Tumor extension (black arrow) beyond the muscularis propria (white arrow) into the serosal fat (black star) can be seen; $\mathrm{H}$ $\&$ E, original magnification $\times 10$.

radiotherapy $(6,10)$. Other studies have also analyzed the impact of extramural spread with respect to the survival rate in patients with neoadjuvant chemotherapy and preoperative radiotherapy $(8,25)$. These studies support the fact, patients with DME $>5 \mathrm{~mm}$ have worse prognosis compared with $\mathrm{DME}<5 \mathrm{~mm}$. In a study of 679 patients with rectal cancer, MERCURY group substantiated DME to be equivalent on MRI and histopathology with a mean difference of less than $0.5 \mathrm{~mm}$ (11), it shows that MRI evaluation is almost as accurate as histopathology examination. A study performed by Shin et al. found no significant difference between T3a and T3b or T3C and T3d with respect to the 5year survival rate but when re-categorized as T3ab (DME < $5 \mathrm{~mm}$ ) and T3cd (DME $<5 \mathrm{~mm}$ ), the 5-year survival rate for T3ab was significantly higher than T3cd (8).

Other studies already mentioned that the subdivision, which we proposed in our study, is important not only for the analysis of treatment and predicting survival but also for selecting proper patients for adjuvant and neoadjuvant treatment $(7,26)$. The clinical guidelines for neoadjuvant and adjuvant therapy vary from country to country. Patients with less than $5 \mathrm{~mm}$ between the tumor border and the mesorectal fascia are given a long-course of preoperative chemo-radiation therapy in some places but also underwent surgery alone in some places (27, 28). Current guidelines recommend preoperative chemoradiation therapy and post-operative chemotherapy for clinically staged T3, T4 (27). Local recurrence and overall survival in rectal cancer have shown significant improvement, but the risk of side effects such as bowel and sexual dysfunction by unnecessary chemotherapy and radiotherapy that is caused by over-staging and risk of local recur- 
rence due to under-staging is also frequently seen (28-30).

\subsection{Limitations of Our Study}

Although MRI is able to provide pre-treatment diagnosis and post treatment evaluation with high accuracy, we still consider pathological result as a gold standard. In this study, we lack confirmation of radiological result with pathologic reference standard for the actual depth of extension (T3 sub-staging), but in our defense, a study revealed high agreement (92\%) up to $0.5 \mathrm{~mm}$ for extramural tumor extension between radiologic and histopathologic diagnosis (11), and this is a reproducibility study rather than accuracy study. Therefore, it should not be criticized from that point of view.

However, our aim was to target the general radiologists who have no specific training in rectal cancer T3 substaging. The radiological classification based on our study i.e. early-staged T3 tumor (T3ab) and advanced-staged T3 tumor (T3cd) decreases the variation of sub-staging result compared to individual measurement (T3a, T3b, T3c and T3d). In the future, more studies should be carried out focusing on the treatment decision influenced by T3 substaging according to the depth of mesorectal invasion extension in rectal cancer using MRI.

In conclusion, the subdivision of T3 stage tumor into early $(<5 \mathrm{~mm})$ and advanced $\mathrm{T} 3$ tumor $(>5 \mathrm{~mm})$ resulted better reproducibility than individually in MRI. This is definitely going to help the non-expert radiologists to determine the accurate treatment method preoperatively by avoiding over- and under-treatment.

\section{Footnotes}

Authors' Contributions: Dr. Tripathi Pratik developed the original idea and the protocol, abstracted and analyzed data, wrote the manuscript. Prof. Mengsu Zeng, Dr. Chun Yang, Dr. Weifeng Guo and Dr. Rai Bimal contributed to the development of the protocol, abstracted data, and prepared the manuscript.

Conflict of Interest Statement: We declare that there are no conflicts of interest in the content of this article.

Financial Disclosure: None declared.

Funding/Support: None declared.

\section{References}

1. Kang H, O'Connell JB, Leonardi MJ, Maggard MA, McGory ML, Ko CY Rare tumors of the colon and rectum: a national review. Int J Colorectal Dis. 2007;22(2):183-9. doi: 10.1007/s00384-006-0145-2. [PubMed: 16845516].
2. Taylor FG, Swift RI, Blomqvist L, Brown G. A systematic approach to the interpretation of preoperative staging MRI for rectal cancer. AJR Am J Roentgenol. 2008;191(6):1827-35. doi: 10.2214/AJR.08.1004. [PubMed: 19020255].

3. Al-Sukhni E, Milot L, Fruitman M, Beyene J,Victor JC, Schmocker S, et al. Diagnostic accuracy of MRI for assessment of T category, lymph node metastases, and circumferential resection margin involvement in patients with rectal cancer: a systematic review and meta-analysis. Ann Surg Oncol. 2012;19(7):2212-23. doi: 10.1245/s10434-011-2210-5. [PubMed: 22271205].

4. Beets-Tan RG. MRI in rectal cancer: the T stage and circumferential resection margin. Colorectal Dis. 2003;5(5):392-5. doi: 10.1046/j.14631318.2003.00518.x. [PubMed: 12925068].

5. Rao SX, Zeng MS, Xu JM, Qin XY, Chen CZ, Li RC, et al. Assessment of $\mathrm{T}$ staging and mesorectal fascia status using high-resolution MRI in rectal cancer with rectal distention. World J Gastroenterol. 2007;13(30):4141-6. doi: 10.3748/wjg.v13.i30.4141. [PubMed: 17696238].

6. Akagi Y, Shirouzu K, Fujita S, Ueno H, Takii Y, Komori K, et al. Predicting oncologic outcomes by stratifying mesorectal extension in patients with pT3 rectal cancer: a Japanese multi-institutional study. Int J Cancer. 2012;131(5):1220-7. doi: 10.1002/ijc.27315. [PubMed: 22025276].

7. Merkel S, Mansmann U, Siassi M, Papadopoulos T, Hohenberger $\mathrm{W}$, Hermanek P. The prognostic inhomogeneity in pT3 rectal carcinomas. Int J Colorectal Dis. 2001;16(5):298-304. doi: 10.1007/s003840100309. [PubMed: 11686527].

8. Shin R, Jeong SY, Yoo HY, Park KJ, Heo SC, Kang GH, et al. Depth of mesorectal extension has prognostic significance in patients with T3 rectal cancer. Dis Colon Rectum. 2012;55(12):1220-8. doi: 10.1097/DCR.ob013e31826fea6a. [PubMed: 23135579].

9. Bori R, Sejben I, Svebis M, Vajda K, Marko L, Pajkos G, et al. Heterogeneity of pT3 colorectal carcinomas according to the depth of invasion. Pathol Oncol Res. 2009;15(3):527-32. doi: 10.1007/s12253-009-9149x. [PubMed: 19172413].

10. Miyoshi M, Ueno H, Hashiguchi Y, Mochizuki H, Talbot IC. Exten of mesorectal tumor invasion as a prognostic factor after curative surgery for T3 rectal cancer patients. Ann Surg. 2006;243(4):492-8. doi 10.1097/01.sla.0000205627.05769.08. [PubMed: 16552200].

11. Mercury Study Group . Extramural depth of tumor invasion at thinsection MR in patients with rectal cancer: results of the MERCURY study. Radiology. 2007;243(1):132-9. doi: 10.1148/radiol.2431051825. [PubMed: 17329685].

12. Gowdra Halappa V, Corona Villalobos CP, Bonekamp S, Gearhart SL, Efron J, Herman J, et al. Rectal imaging: part 1, High-resolution MRI of carcinoma of the rectum at 3 T. AJR Am J Roentgenol. 2012;199(1):W3542. doi: 10.2214/AJR.11.8134. [PubMed: 22733930].

13. Spiegle G, Leon-Carlyle M, Schmocker S, Fruitman M, Milot L, Gagliardi AR, et al. Development of a synoptic MRI report for primary rectal cancer. Implement Sci. 2009;4:79. doi:10.1186/1748-5908-4-79. [PubMed: 19954526].

14. Shirouzu K, Akagi Y, Fujita S, Ueno H, Takii Y, Komori K, et al. Clinical significance of the mesorectal extension of rectal cancer: a Japanese multi-institutional study. Ann Surg. 2011;253(4):704-10. doi: 10.1097/SLA.ob013e3182119331. [PubMed: 21475010].

15. Shrout PE, Fleiss JL. Intraclass correlations: uses in assessing rater reliability. Psychol Bull.1979;86(2):420-8. doi: 10.1037|0033-2909.86.2.420. [PubMed: 18839484].

16. Hallgren KA. Computing Inter-Rater Reliability for Observational Data: An Overview and Tutorial. Tutor Quant Methods Psychol. 2012;8(1):23-34. doi: 10.20982/tqmp.08.1.p023. [PubMed: 22833776].

17. Egner JR. AJCC cancer staging manual. 304. JAMA: The Journal of the American Medical Association; 2010.1726 p.

18. Kaur H, Choi H, You YN, Rauch GM, Jensen CT, Hou P, et al. MR imaging for preoperative evaluation of primary rectal cancer: practical considerations. Radiographics. 2012;32(2):389-409. doi: 10.1148/rg.322115122. [PubMed: 22411939]. 
19. Pedersen BG, Moran B, Brown G, Blomqvist L, Fenger-Gron M, Laurberg S. Reproducibility of depth of extramural tumor spread and distance to circumferential resection margin at rectal MRI: enhancement of clinical guidelines for neoadjuvant therapy. AJR Am J Roentgenol. 2011;197(6):1360-6. doi: 10.2214/AJR.11.6508. [PubMed: 22109290].

20. Cho SH, Kim SH, Bae JH, Jang YJ, Kim HJ, Lee D, et al. Prognostic stratification by extramural depth of tumor invasion of primary rectal cancer based on the Radiological Society of North America proposal. AJR Am J Roentgenol. 2014;202(6):1238-44. doi: 10.2214/AJR.13.11311. [PubMed: 24848820].

21. Glimelius B, Pahlman L, Cervantes A, Esmo Guidelines Working Group . Rectal cancer: ESMO Clinical Practice Guidelines for diagnosis, treatment and follow-up. Ann Oncol. 2010;21 Suppl 5:v82-6. doi:10.1093/annonc/mdq170. [PubMed: 20555109].

22. Benson A3, Bekaii-Saab T, Chan E, Chen YJ, Choti MA, Cooper HS, et al. Rectal cancer. J Natl Compr Canc Netw. 2012;10(12):1528-64. [PubMed: 23221790].

23. Taylor FG, Quirke P, Heald RJ, Moran B, Blomqvist L, Swift I, et al. Preoperative high-resolution magnetic resonance imaging can identify good prognosis stage I, II, and III rectal cancer best managed by surgery alone: a prospective, multicenter, European study. Ann Surg. 2011;253(4):711-9. doi: 10.1097/SLA.0b013e31820b8d52. [PubMed: 21475011].

24. Schmoll HJ, Van Cutsem E, Stein A, Valentini V, Glimelius B, Haustermans K, et al. ESMO Consensus Guidelines for management of patients with colon and rectal cancer. a personalized approach to clinical decision making. Ann Oncol. 2012;23(10):2479-516. doi: 10.1093/an- nonc/mds236. [PubMed: 23012255].

25. Merkel S, Weber K, Schellerer V, Gohl J, Fietkau R, Agaimy A, et al. Prognostic subdivision of ypT3 rectal tumours according to extension beyond the muscularis propria. BrJ Surg. 2014;101(5):566-72. doi: 10.1002/bjs.9419. [PubMed: 24477831].

26. Zinicola R, Pedrazzi G, Haboubi N, Nicholls RJ. The degree of extramural spread of T3 rectal cancer: an appeal to the American Joint Committee on Cancer. Colorectal Dis. 2017;19(1):8-15. doi: 10.1111/codi.13565. [PubMed: 27883254].

27. Benson A3, Venook AP, Bekaii-Saab T, Chan E, Chen YJ, Cooper HS, et al. Rectal Cancer, Version 2.2015. J Natl Compr Canc Netw. 2015;13(6):71928. quiz 728. doi: 10.6004/jnccn.2015.0087. [PubMed: 26085388].

28. Breugom AJ, Vermeer TA, van den Broek CB, Vuong T, Bastiaannet E, Azoulay L, et al. Effect of preoperative treatment strategies on the outcome of patients with clinical T3, non-metastasized rectal cancer: A comparison between Dutch and Canadian expert centers. Eur J Surg Oncol. 2015;41(8):1039-44. doi: 10.1016/j.ejso.2015.05.002. [PubMed: 26059312].

29. Marijnen CA, Kapiteijn E, van de Velde CJ, Martijn H, Steup WH, Wiggers $\mathrm{T}$, et al. Acute side effects and complications after short-term preoperative radiotherapy combined with total mesorectal excision in primary rectal cancer: report of a multicenter randomized trial.J Clin Oncol.2002;20(3):817-25. doi:10.1200/JCO.2002.20.3.817.[PubMed: 11821466].

30. Lange MM, Marijnen CA, Maas CP, Putter H, Rutten HJ, Stiggelbout $\mathrm{AM}$, et al. Risk factors for sexual dysfunction after rectal cancer treatment. Eur J Cancer. 2009;45(9):1578-88. doi: 10.1016/j.ejca.2008.12.014. [PubMed: 19147343]. 